\title{
NEW COINCIDENCE POINT THEOREMS IN CONTINUOUS FUNCTION SPACES AND APPLICATIONS
}

\author{
JUN WU ${ }^{\otimes}$ and YICHENG LIU
}

(Received 28 April 2008)

\begin{abstract}
In this paper, some new coincidence point theorems in continuous function spaces are presented. We show the hybrid mapping version and multivalued version of both Lou's fixed point theorem (Proc. Amer. Math. Soc. 127 (1999)) and de Pascale and de Pascale's fixed point theorem (Proc. Amer. Math. Soc. 130 (2002)). Our new results encompass a number of previously known generalizations of the theorems. Two examples are presented.
\end{abstract}

2000 Mathematics subject classification: primary 47H10; secondary 54H25.

Keywords and phrases: fixed point theorem, multivalued mapping, coincidence point.

\section{Introduction}

Let $E$ be a Banach space equipped with the norm $\|\cdot\|_{E}, I=[0, T]$ for $T>0$. Here $C(I, E)$ denotes the Banach space consisting of all continuous mappings from $I$ into $E$ with norm $\|u\|_{C}=\max \left\{\|u(t)\|_{E}: t \in[0, T]\right\}$ for $u \in C(I, E)$. In 1999, Lou [5, Corollary 2.1] proved a fixed point theorem in continuous function spaces. Using the notion of $K$-normed spaces, de Pascale and de Pascale in [2, Corollary 2.2] proved a similar fixed point theorem. Then, de Pascale and Zabreiko gave a generalization result in [3]. Recently, Suzuki [6] presented simple proofs for the above theorems.

In this paper, we present some new coincidence point theorems in continuous function spaces. These results have both a hybrid mapping version and a multivalued version of the previous fixed point theorems in [2, 5] and encompass a number of previously known generalizations of the theorems in $[1,5,6]$. In the final section, two examples are presented to illustrate the effectiveness of our results.

The work of J. Wu has been partially supported by the Scientific Research Fund of Hunan Provincial Education Department (08C117) and the Scientific Research Fund for the Doctoral Program of CSUST (1004132).

(C) 2009 Australian Mathematical Society 0004-9727/2009 \$16.00 


\section{Main results}

In this section, we show two generalizations of Lou's fixed point theorem in [5] and de Pascale and de Pascale's result in [2]. First, we give a coincidence point theorem for two self-mappings in continuous function spaces.

\subsection{Hybrid-map version for Lou's fixed point theorem}

THEOREM 2.1. Let $F$ be a nonempty closed subset of $C(I, E)$ and $A, B: F \rightarrow F$ two operators. If there exist $\alpha, \beta \in[0,1), K \geq 0$ such that for any $u, v \in F$ and $t \in I \backslash\{0\}$,

$$
\|A u(t)-A v(t)\|_{E} \leq \beta\|B u(t)-B v(t)\|_{E}+\frac{K}{t^{\alpha}} \int_{0}^{t}\|B u(s)-B v(s)\|_{E} d s .
$$

If $B F$ is a closed subset of $F$ and $A F \subset B F$, then $A$ and $B$ have a coincidence point. Furthermore, if $A$ or $B$ is injective and $A B=B A$, then $A$ and $B$ have a unique common fixed point.

PRoof. For any given $x_{0} \in F$, set $y_{0}=B x_{0}$, since $A F \subset B F$, there is $x_{1} \in F$ such that $B x_{1}=A x_{0}$, and set $y_{1}=B x_{1}$. For $x_{1}$, there is $x_{2} \in F$ such that $y_{2}=B x_{2}=A x_{1}$. We continue this process to obtain a sequence $\left\{y_{n}\right\}$ in $F$ such that

$$
x_{n} \in F \quad \text { and } \quad y_{n}=B x_{n}=A x_{n-1}, \quad n=1,2, \ldots
$$

Using the equivalent norm method [6], we prove that the sequence $\left\{y_{n}\right\}$ is a Cauchy sequence with respect to norm $\|\cdot\|_{C}$. First, we choose a $\tau \in(0, T]$ such that

$$
\beta+K \tau^{1-\alpha}<1
$$

and define a decreasing function $f$ from $I$ into $(0,1]$ by

$$
f(t)= \begin{cases}1 & \text { if } t \in(0, \tau], \\ e^{1-t / \tau} & \text { if } t \in(\tau, T] .\end{cases}
$$

Define another norm $\|\cdot\|_{1}$ in $C(I, E)$ by

$$
\|u\|_{1}=\max \left\{f(t)\|u(t)\|_{E}: t \in I\right\} \quad \forall u \in C(I, E) .
$$

Then the two norms $\|\cdot\|_{C}$ and $\|\cdot\|_{1}$ are equivalent.

Next, we prove that $\left\{y_{n}\right\}$ is a Cauchy sequence with respect to the norm $\|\cdot\|_{1}$. Set

$$
a_{n}(t)=\left\|y_{n+1}(t)-y_{n}(t)\right\|_{E} \quad \text { and } \quad a_{n}=\left\|y_{n+1}-y_{n}\right\|_{1} .
$$

Following (2.1) and (2.2),

$$
a_{n+1}(t) \leq \beta a_{n}(t)+\frac{K}{t^{\alpha}} \int_{0}^{t} a_{n}(s) d s .
$$


If $t \in(0, \tau]$, since $a_{n}(t) \leq a_{n}$,

$$
f(t) a_{n+1}(t)=a_{n+1}(t) \leq \beta a_{n}+K \tau^{1-\alpha} a_{n}=\left(\beta+K \tau^{1-\alpha}\right) a_{n} .
$$

If $t \in(\tau, T]$, since $a_{n}(t) \leq e^{-1+t / \tau} a_{n}$,

$$
\int_{0}^{t} a_{n}(s) d s=\int_{0}^{\tau} a_{n}(s) d s+\int_{\tau}^{t} a_{n}(s) d s \leq \tau e^{-1+t / \tau} a_{n} .
$$

Thus,

$$
f(t) a_{n+1}(t)=e^{1-t / \tau} a_{n+1}(t) \leq\left(\beta+K \tau^{1-\alpha}\right) a_{n} .
$$

Then, for $t \in(0, T]$,

$$
f(t) a_{n+1}(t) \leq\left(\beta+K \tau^{1-\alpha}\right) a_{n} .
$$

This implies $a_{n+1} \leq\left(\beta+K \tau^{1-\alpha}\right) a_{n}$. So

$$
a_{n+1} \leq\left(\beta+K \tau^{1-\alpha}\right)^{n+1} a_{0} .
$$

Then $\left\{y_{n}\right\}$ is a Cauchy sequence with respect to the norm $\|\cdot\|_{1}$ for $\beta+K \tau^{1-\alpha}<1$. Thus, $\left\{y_{n}\right\}$ is a Cauchy sequence with respect to the norm $\|\cdot\|_{C}$. Then there is a point $y \in F$ such that

$$
\lim _{n \rightarrow \infty} A x_{n}=\lim _{n \rightarrow \infty} B x_{n}=y .
$$

Since $B F$ is a closed subset of $F$ and $y \in B F$, then there exists a $u \in F$ such that $y=B u$. We claim that $y=A u$. In fact, by (2.1),

$$
\begin{aligned}
\left\|A u(t)-A x_{n}(t)\right\|_{E} & \leq \beta\left\|B u(t)-B x_{n}(t)\right\|_{E}+\frac{K}{t^{\alpha}} \int_{0}^{t}\left\|B u(s)-B x_{n}(s)\right\|_{E} d s \\
& =\beta\left\|y(t)-B x_{n}(t)\right\|_{E}+\frac{K}{t^{\alpha}} \int_{0}^{t}\left\|y(s)-B x_{n}(s)\right\|_{E} d s \\
& \leq\left(\beta+K T^{1-\alpha}\right)\left\|y-B x_{n}\right\|_{C} .
\end{aligned}
$$

Thus, $y=A u$ and $u$ is a coincidence point of $A$ and $B$.

Now we prove the uniqueness of the coincidence point. Without loss of generality, we suppose that $B$ is injective. If both $u$ and $v(u \neq v)$ are the coincidence points of $A$ and $B$, then $A u=B u, A v=B v$ and $B u \neq B v$. Following (2.1),

$$
\|A u(t)-A v(t)\|_{E} \leq \beta\|B u(t)-B v(t)\|_{E}+\frac{K}{t^{\alpha}} \int_{0}^{t}\|B u(s)-B v(s)\|_{E} d s .
$$

Based on the above arguments, it is easy to see that

$$
\|B u-B v\|_{1}=\|A u-A v\|_{1} \leq\left(\beta+K \tau^{1-\alpha}\right)\|B u-B v\|_{1} .
$$

This is impossible. Thus, the coincidence point of $A$ and $B$ is unique. 
Noting that $y=A u=B u$ and $A B=B A$,

$$
A y=A B u=B A u=B y .
$$

Thus $y$ is also the coincidence point of $A$ and $B$. Then $y=u$ for the uniqueness. This means

$$
u=A u=B u \text {. }
$$

So $u$ is the unique common fixed point of $A$ and $B$. This completes the proof of Theorem 2.1.

Let $I=[\eta, \infty)$ for $\eta>0, B C(I, E)$ be a Banach space consisting of all bounded continuous mappings from $I$ into $E$ with the norm $\|u\|_{B}=\max \left\{\|u(t)\|_{E}: t \in[\eta, \infty)\right\}$ for $u \in B C(I, E)$. In a similar way to Theorem 2.1, we have the following result.

THEOREM 2.2. Let $F$ be a nonempty closed subset of $B C(I, E)$ and $A, B: F \rightarrow F$ two operators. If there exist $\alpha \in(1, \infty), \beta \in[0,1), K \geq 0$ such that for any $u, v \in F$ and $t \in I$,

$$
\|A u(t)-A v(t)\|_{E} \leq \beta\|B u(t)-B v(t)\|_{E}+\frac{K}{t^{\alpha}} \int_{\eta}^{t}\|B u(s)-B v(s)\|_{E} d s .
$$

If $B F$ is a closed subset of $F$ and $A F \subset B F$, then $A$ and $B$ have a coincidence point. Furthermore, if $A$ or $B$ is injective and $A B=B A$, then $A$ and $B$ have a unique common fixed point.

ProOF. Following the proof of Theorem 2.1, we choose a sequence $\left\{y_{n}\right\}$ in $F$ such that

$$
x_{n} \in F \quad \text { and } \quad y_{n}=B x_{n}=A x_{n-1}, \quad n=1,2, \ldots
$$

Next, we choose two constants $\tau \geq \eta$ and $c>0$ such that

$$
\beta+\frac{K}{c \eta^{\alpha}}+K \tau^{1-\alpha}<1
$$

and define a nonincreasing function $f_{1}$ from $I$ into $(0,1]$ by

$$
f_{1}(t)= \begin{cases}e^{-c t} & \text { if } t \in(\eta, \tau] \\ e^{-c \tau} & \text { if } t \in(\tau, \infty)\end{cases}
$$

Define another norm $\|\cdot\|_{2}$ in $B C(I, E)$ by

$$
\|u\|_{2}=\max \left\{f_{1}(t)\|u(t)\|_{E}: t \in I\right\} \quad \forall u \in B C(I, E) .
$$

Then the two norms $\|\cdot\|_{B}$ and $\|\cdot\|_{2}$ are equivalent.

Set

$$
a_{n}(t)=\left\|y_{n+1}(t)-y_{n}(t)\right\|_{E} \quad \text { and } \quad a_{n}=\left\|y_{n+1}-y_{n}\right\|_{2} \text {, }
$$


then we have

$$
a_{n+1}(t) \leq \beta a_{n}(t)+\frac{K}{t^{\alpha}} \int_{\eta}^{t} a_{n}(s) d s .
$$

If $t \in(\eta, \tau]$, since $a_{n}(t) \leq e^{c t} a_{n}$,

$$
\int_{\eta}^{t} a_{n}(s) d s \leq \int_{\eta}^{t} e^{c s} a_{n} d s \leq \frac{e^{c t}}{c} a_{n} .
$$

Thus,

$$
f_{1}(t) a_{n+1}(t)=e^{-c t} a_{n+1}(t) \leq \beta a_{n}+\frac{K}{c \eta^{\alpha}} a_{n}=\left(\beta+\frac{K}{c \eta^{\alpha}}\right) a_{n} .
$$

If $t \in(\tau, \infty)$, since $a_{n}(t) \leq e^{c \tau} a_{n}$,

$$
\int_{\eta}^{t} a_{n}(s) d s=\int_{\eta}^{\tau} a_{n}(s) d s+\int_{\tau}^{t} a_{n}(s) d s \leq\left(\frac{1}{c}+t\right) e^{c \tau} a_{n} .
$$

Thus,

$$
\begin{aligned}
f_{1}(t) a_{n+1}(t) & =e^{-c \tau} a_{n+1}(t) \leq e^{-c \tau}\left(\beta a_{n}(t)+\frac{K}{t^{\alpha}} \int_{\eta}^{t} a_{n}(s) d s\right) \\
& \leq\left(\beta+\frac{K}{c \eta^{\alpha}}+K \tau^{1-\alpha}\right) a_{n} .
\end{aligned}
$$

Then, for $t \in[\eta, \infty)$,

$$
f_{1}(t) a_{n+1}(t) \leq\left(\beta+\frac{K}{c \eta^{\alpha}}+K \tau^{1-\alpha}\right) a_{n} .
$$

This implies $a_{n+1} \leq\left(\beta+K / c \eta^{\alpha}+K \tau^{1-\alpha}\right) a_{n}$. So

$$
a_{n+1} \leq\left(\beta+\frac{K}{c \eta^{\alpha}}+K \tau^{1-\alpha}\right)^{n+1} a_{0} .
$$

Thus, $\left\{y_{n}\right\}$ is a Cauchy sequence with respect to the norm $\|\cdot\|_{2}$ for $\beta+K / c \eta^{\alpha}+$ $K \tau^{1-\alpha}<1$. Then $\left\{y_{n}\right\}$ is a Cauchy sequence with respect to the norm $\|\cdot\|_{B}$. Therefore, there is a point $y \in F$ such that

$$
\lim _{n \rightarrow \infty} A x_{n}=\lim _{n \rightarrow \infty} B x_{n}=y .
$$

Similarly, we have $y \in B F$ and there exists a $u \in F$ such that $y=B u$. We prove $y=A u$. In fact, by (2.3),

$$
\left\|A u(t)-A x_{n}(t)\right\|_{E} \leq \beta\left\|B u(t)-B x_{n}(t)\right\|_{E}+\frac{K}{t^{\alpha}} \int_{\eta}^{t}\left\|B u(s)-B x_{n}(s)\right\|_{E} d s .
$$


This implies

$$
\left\|A u-A x_{n}\right\|_{B} \leq\left(\beta+\frac{K}{\eta^{\alpha-1}}\right)\left\|y-B x_{n}\right\|_{B} .
$$

Thus, $y=A u$ and $u$ is a coincidence point of $A$ and $B$.

A similar argument to that used in Theorem 2.1 shows the existence and uniqueness of common fixed point of $A$ and $B$. This completes the proof of Theorem 2.2.

In order to investigate the existence of solutions of functional differential equations by using fixed point theorems in continuous function spaces, we consider the Banach space $C[[-\sigma, 0], E]$ for some $\sigma>0$ with supremum norm. For $I=[0, T]$ and $\varphi \in C[[-\sigma, 0], E]$, define a closed subset as $F=\{u \in C[I, E] \mid u(0)=\varphi(0)\}$. Then following similar arguments as in Theorem 2.1, we obtain a useful result. We omit the details of the proof.

TheOREM 2.3. Let $F$ be given as above, $A: F \rightarrow F$ be an operator. For any $u, v \in F$ and $t \in[-\sigma, 0]$, define $u(t)=v(t)=\varphi(t)$, if there exist $\alpha, \beta \in[0,1), K \geq 0$ such that for $t \in I \backslash\{0\}$,

$$
\begin{array}{r}
\|A u(t)-A v(t)\|_{E} \leq \beta \sup _{s \in[-\sigma, 0]}\left\{\|u(t+s)-v(t+s)\|_{E}\right\} \\
+\frac{K}{t^{\alpha}} \int_{0}^{t}\|u(s)-v(s)\|_{E} d s .
\end{array}
$$

Then A has a unique fixed point.

Corollary 2.4 (Lou [5]). Let $I=[0, T], F$ be a nonempty closed subset of $C(I, E)$ and $A: F \rightarrow F$ an operator. If there exist $\alpha, \beta \in[0,1), K \geq 0$ such that for any $u, v \in F$ and $t \in I \backslash\{0\}$,

$$
\|A u(t)-A v(t)\|_{E} \leq \beta\|u(t)-v(t)\|_{E}+\frac{K}{t^{\alpha}} \int_{0}^{t}\|u(s)-v(s)\|_{E} d s .
$$

Then A has a unique fixed point.

Corollary 2.5 (de Pascale and de Pascale [2]). Let $I=[\eta, \infty)$, F be a nonempty closed subset of $B C(I, E)$ and $A: F \rightarrow F$ an operator. If there exist $\alpha \in[1, \infty)$, $\beta \in[0,1), K \geq 0$ such that for any $u, v \in F$ and $t \in I$,

$$
\|A u(t)-A v(t)\|_{E} \leq \beta\|u(t)-v(t)\|_{E}+\frac{K}{t^{\alpha}} \int_{\eta}^{t}\|u(s)-v(s)\|_{E} d s .
$$

Then A has a unique fixed point.

2.2. Multivalued version for Lou's fixed point theorem Let $(X, d)$ be a metric space, for $x \in X, A \subset X$, define $D(x, A)=\inf \{d(x, y), y \in A\}$. We denote $C B(X)$ as the class of all nonempty bounded closed subsets of $X$. Let $H$ be the Hausdorff metric with respect to $d$, that is, $H(A, B)=\max \left\{\sup _{x \in A} D(x, B), \sup _{y \in B} D(y, A)\right\}$ 
for every $A, B \in C B(X)$. Then we have the following multivalued coincidence point theorem, which can be regarded to some extent as a multivalued version of Lou's theorem.

THEOREM 2.6. For $I=[0, T]$, let $F$ be a nonempty closed subset of $C(I, E), A$ : $F \rightarrow C B(F)$ a multivalued operator and $B: F \rightarrow F$ a single-valued operator. If there exist $\alpha, \beta \in[0,1), K \geq 0$ such that for any $u, v \in F$ and $t \in I \backslash\{0\}$,

$$
H(A u, A v) \leq \beta\|B u(t)-B v(t)\|_{E}+\frac{K}{t^{\alpha}} \int_{0}^{t}\|B u(s)-B v(s)\|_{E} d s .
$$

If $B F$ is a closed subset of $F$ and $A F \subset B F$, then $A$ and $B$ have a coincidence point.

Proof. First, we choose a $\tau \in(0, T]$ such that $\beta+K \tau^{1-\alpha}<1$. Set $\lambda=\beta+K \tau^{1-\alpha}$. Following the method in [4], we construct a fixed point iteration sequence in $F$. For any given $x_{0} \in F$, by virtue of $A F \subset B F$, there is an $x_{1} \in F$ such that $y_{1}=$ $B x_{1} \in A x_{0}$. Since $A x_{0}$ and $A x_{1}$ are closed sets and $y_{1} \in A x_{0}$, we can find $y_{2} \in A x_{1}$ such that

$$
\left\|y_{1}-y_{2}\right\|_{C} \leq H\left(A x_{0}, A x_{1}\right)+\lambda .
$$

Using $A F \subset B F$ again, for $x_{1} \in F$, there exists an $x_{2}$ such that $B x_{2}=y_{2} \in A x_{1}$, then we choose $y_{3} \in A x_{2}$ satisfying

$$
\left\|y_{2}-y_{3}\right\|_{C} \leq H\left(A x_{1}, A x_{2}\right)+\lambda^{2},
$$

and $y_{3}=B x_{3}$ for some $x_{3} \in F$.

We continue this process to obtain a sequence $\left\{y_{n}\right\}$ in $F$ such that

$$
\begin{gathered}
y_{n}=B x_{n} \in A x_{n-1} \quad \text { and }\left\|y_{n+1}-y_{n}\right\|_{C} \leq H\left(A x_{n-1}, A x_{n}\right)+\lambda^{n}, \\
n=1,2, \ldots
\end{gathered}
$$

Let $b_{n}=\left\|y_{n}-y_{n+1}\right\|_{1}$ and $b_{n}(t)=\left\|y_{n}(t)-y_{n+1}(t)\right\|_{E}$, where $\|\cdot\|_{1}$ has been defined in the proof of Theorem 2.1, then

$$
\begin{aligned}
\left\|y_{n+2}-y_{n+1}\right\|_{C} & \leq H\left(A x_{n+1}, A x_{n}\right)+\lambda^{n+1} \\
& \leq \beta b_{n}(t)+\frac{K}{t^{\alpha}} \int_{0}^{t} b_{n}(s) d s+\lambda^{n+1} .
\end{aligned}
$$

If $t \in(0, \tau]$, since $b_{n}(t) \leq b_{n}$,

$$
f(t)\left\|y_{n+2}-y_{n+1}\right\|_{C}=\left\|y_{n+2}-y_{n+1}\right\|_{C} \leq\left(\beta+K \tau^{1-\alpha}\right) b_{n}+\lambda^{n+1} .
$$

If $t \in(\tau, T]$, since $b_{n}(t) \leq e^{-1+t / \tau} b_{n}$,

$$
\int_{0}^{t} b_{n}(s) d s=\int_{0}^{\tau} b_{n}(s) d s+\int_{\tau}^{t} b_{n}(s) d s \leq \tau e^{-1+t / \tau} b_{n} .
$$


Thus,

$$
f(t)\left\|y_{n+2}-y_{n+1}\right\|_{C}=e^{1-t / \tau}\left\|y_{n+2}-y_{n+1}\right\|_{C} \leq\left(\beta+K \tau^{1-\alpha}\right) b_{n}+\lambda^{n+1} .
$$

Then, for $t \in(0, T]$,

$$
f(t)\left\|y_{n+2}-y_{n+1}\right\|_{C} \leq\left(\beta+K \tau^{1-\alpha}\right) b_{n}+\lambda^{n+1} .
$$

This implies

$$
b_{n+1} \leq\left(\beta+K \tau^{1-\alpha}\right) b_{n}+\lambda^{n+1}=\lambda b_{n}+\lambda^{n+1} .
$$

So

$$
b_{n+1} \leq \lambda^{n+1}\left(b_{0}+n+1\right) \quad \forall n=0,1,2, \ldots
$$

It is easy to show $\lim _{n \rightarrow \infty} b_{n}=0$.

For any $\varepsilon>0$, we choose a sufficiently large number $N$ such that

$$
\lambda^{N}\left(b_{0}+N\right) \leq \frac{\varepsilon(1-\lambda)}{2}, \quad \lambda^{N} \leq \frac{\varepsilon(1-\lambda)^{2}}{4} .
$$

Thus, for any positive integer $k$, we obtain

$$
\begin{aligned}
\left\|y_{N}-y_{N+k}\right\|_{1} & \leq \sum_{i=0}^{k-1} b_{N+i} \leq \sum_{i=0}^{k-1} \lambda^{N+i}\left(b_{0}+N+i\right) \\
& <\frac{\lambda^{N}\left(b_{0}+N\right)}{1-\lambda}+\lambda^{N} \sum_{i=0}^{k-1} i \lambda^{i} \\
& <\frac{\lambda^{N}\left(b_{0}+N\right)}{1-\lambda}+\frac{2 \lambda^{N}}{(1-\lambda)^{2}} \leq \varepsilon
\end{aligned}
$$

This means that $\left\{y_{n}\right\}$ is a Cauchy sequence with respect to the norm $\|\cdot\|_{1}$. Thus, $\left\{y_{n}\right\}$ is a Cauchy sequence with respect to the norm $\|\cdot\|_{C}$.

Let $\lim _{n \rightarrow \infty} y_{n}=y \in F$. By virtue of $B F$ being a closed set, we have $y=B u$ for some $u \in F$. We claim that $B u \in A u$. Indeed, condition (2.5) implies that

$$
\begin{aligned}
H\left(A u, A x_{n}\right) & \leq \beta\left\|B u(t)-y_{n}(t)\right\|_{E}+\frac{K}{t^{\alpha}} \int_{0}^{t}\left\|B u(s)-y_{n}(s)\right\|_{E} d s \\
& \leq\left(\beta+K T^{1-\alpha}\right)\left\|B u-y_{n}\right\|_{C} .
\end{aligned}
$$

Since $y_{n} \in A x_{n-1}$, we obtain

$$
D\left(A u, y_{n+1}\right) \leq H\left(A u, A x_{n}\right) \leq\left(\beta+K T^{1-\alpha}\right)\left\|B u-y_{n}\right\|_{C} .
$$

Thus, $D(A u, y)=0$. This means $B u=y \in A u$. Hence, $A$ and $B$ have a coincidence point $u$. This completes the proof. 
Using the same technique as in Theorems 2.2 and 2.6, we obtain the following multivalued coincidence point theorem.

THEOREM 2.7. For $I=[\eta, \infty)$ and $\eta>0$, let $F$ be a nonempty closed subset of $B C(I, E), A: F \rightarrow C B(F)$ a multivalued operator and $B: F \rightarrow F$ is a single-valued operator. If there exist $\alpha>1, \beta \in[0,1), K \geq 0$ such that for any $u, v \in F$ and $t \in I$,

$$
H(A u, A v) \leq \beta\|B u(t)-B v(t)\|_{E}+\frac{K}{t^{\alpha}} \int_{\eta}^{t}\|B u(s)-B v(s)\|_{E} d s .
$$

If $B F$ is closed subset of $F$ and $A F \subset B F$, then $A$ and $B$ have a coincidence point.

Let $B=\left.I d\right|_{F}$ in Theorem 2.6, we obtain a generalization of the multivalued contraction principle established by Covitz in [1].

Corollary 2.8. Let $F$ be a nonempty closed subset of $C(I, E)$ and $A: F$ $\rightarrow C B(F)$ a multivalued operator. If there exist $\alpha, \beta \in[0,1), K \geq 0$ such that for any $u, v \in F$ and $t \in I \backslash\{0\}$,

$$
H(A u, A v) \leq \beta\|u(t)-v(t)\|_{E}+\frac{K}{t^{\alpha}} \int_{0}^{t}\|u(s)-v(s)\|_{E} d s .
$$

Then A has a fixed point.

\section{Some applications}

EXAMPLE 3.1. Consider the existence of solutions of the following differential equations with distributed delay:

$$
\left[x(t)-\int_{-\tau}^{0} k(s) x(t+s) d s\right]^{\prime}=f\left(t, \int_{0}^{t} h(t, s) x(s) d s\right),
$$

where $t \in I=[0, T]$ for $T>0, \quad \tau>0, \quad f \in C\left[I \times R^{n}, R^{n}\right], \quad h \in C\left[\Omega, R^{n \times n}\right]$, $\Omega=\left\{(t, s) \in I^{2} \mid 0 \leq s \leq t \leq T\right\}$ and $k \in C\left[I, R^{n \times n}\right]$ with $\int_{-\tau}^{0}|k(s)| d s=k<1$. $x(t)=\varphi(t)$ for $t \in[-\tau, 0]$ and $\varphi \in C\left[[-\tau, 0], R^{n}\right]$.

( $\left.F_{1}\right)$ There exists a constant $R$ such that, for any $u, v \in R^{n}$,

$$
|f(t, u)-f(t, v)| \leq R|u-v| .
$$

THEOREM 3.2. Suppose that $\left(F_{1}\right)$ holds, then (3.1) has a unique solution on I.

PROOF. We transfer the existence of solutions of (3.1) into a fixed point problem. Let $F=\left\{u \in C\left[I, R^{n}\right] \mid u(0)=\varphi(0)\right\}$, define $A: F \rightarrow F$ by

$$
\begin{aligned}
A u(t)=\varphi(0) & -\int_{-\tau}^{0} k(s) \varphi(s) d s+\int_{-\tau}^{0} k(s) u(t+s) d s \\
& +\int_{0}^{t} f\left(s, \int_{0}^{s} h(s, r) u(r) d r\right) d s
\end{aligned}
$$


where $u(t)=\varphi(t)$ for $t \in[-\tau, 0]$. Then (3.1) has the solution

$$
S(t)= \begin{cases}u(t) & \text { if } t \in I \\ \varphi(t) & \text { if } t \in[-\tau, 0]\end{cases}
$$

if and only if $u(t)$ is the fixed point of $A$. Furthermore, by direct computation,

$$
|A u(t)-A v(t)| \leq k \sup _{s \in[-\tau, 0]}\{|u(t+s)-v(t+s)|\}+R h \int_{0}^{t}|u(s)-v(s)| d s,
$$

where $h=\max \{|h(t, s)|:(t, s) \in \Omega\} . \quad$ Take $\alpha=0, \quad \beta=k$ and $K=R h$, then Theorem 2.3 shows that $A$ has exactly one fixed point $u$ in $C\left[I, R^{n}\right]$. Thus, (3.1) has a unique solution $S(t)$. This completes the proof.

Next we consider an example presented by Lou in [5] to see that one of the restrictions in [5] is not necessary.

EXAMPLE 3.3. Consider the integro-differential equation of mixed type:

$$
x^{\prime}(t)=f(t, x, T x, S x), \quad t \in I=[0,1], x(0)=x_{0},
$$

where $f \in C\left[I \times R^{1} \times R^{1} \times R^{1}, R^{1}\right], x_{0} \in R^{1}$ and

$$
T x(t)=\int_{0}^{t} k(t, s) x(s) d s, \quad S x(t)=\int_{0}^{1} h(t, s) x(s) d s
$$

with $k \in C\left[\Omega, R_{+}\right], \Omega=\left\{(t, s) \in I^{2} \mid 0 \leq s \leq t \leq 1\right\}, h \in C\left[I \times I, R_{+}\right]$.

First, we establish the existence of minimal and maximal solution of problem (3.2) and list Lou's conditions [5] as follows.

$\left(H_{1}\right)$ There exist $p, q \in C\left[I, R^{1}\right], p(t) \leq q(t)(t \in I)$ such that

$$
p^{\prime} \leq f(t, p, T p, S p), \quad p(0) \leq x_{0} ; \quad q^{\prime} \geq f(t, q, T q, S q), \quad q(0) \geq x_{0} .
$$

$\left(H_{2}\right)$ There exist $M>0$ and $Q, R \geq 0$ such that

$$
f(t, x, u, v)-f(t, \bar{x}, \bar{u}, \bar{v}) \leq-M(x-\bar{x})-R(u-\bar{u})-Q(v-\bar{v})
$$

for $t \in I, \quad p(t) \leq \bar{x} \leq x \leq q(t), \quad T p(t) \leq \bar{u} \leq u \leq T q(t)$ and $\quad S p(t) \leq \bar{v} \leq v$ $\leq S q(t)$.

TheOREM 3.4. Suppose that $\left(H_{1}\right)$ and $\left(H_{2}\right)$ hold. Then there exist monotone sequences $\left\{p_{n}\right\},\left\{q_{n}\right\} \subset C^{1}\left[I, R^{1}\right]$ such that

$$
p(t)=p_{0}(t) \leq p_{1}(t) \leq \cdots \leq p_{n}(t) \leq \cdots \leq q_{n}(t) \leq \cdots \leq q_{1}(t) \leq q_{0}(t)=q(t)
$$

and $p_{n}(t) \rightarrow x_{*}(t), q_{n}(t) \rightarrow x^{*}(t)$ as $n \rightarrow \infty$ uniformly in $t \in I, x_{*}, x^{*} \in C^{1}\left[I, R^{1}\right]$. Moreover, $x_{*}$ and $x^{*}$ are the minimal and maximal solutions of (3.2) on the interval $[p, q]$, respectively. 
Proof. Set $U=\left\{\eta \in C\left[I, R^{1}\right] \mid p \leq \eta \leq q\right\}$. For any $\eta \in U$, consider the linear equation

$$
x^{\prime}(t)=\sigma(t)-M x(t)-R T x(t)-Q S x(t), \quad x(0)=x_{0},
$$

where $\sigma(t)=f(t, \eta(t), T \eta(t), S \eta(t))+M \eta(t)+R T \eta(t)+Q S \eta(t)$. Then $x$ $\in C^{1}\left[I, R^{1}\right]$ is a solution of (3.3) if and only if $x \in C\left[I, R^{1}\right]$ is a solution of integral equation

$$
x(t)=e^{-M t}\left\{x_{0}+\int_{0}^{t} e^{M s}(\sigma(s)-R T x(s)-Q S x(s)) d s\right\} \equiv A x(t) .
$$

Noting that both $T$ and $S$ are linear bounded operators, we see that, for any $x$, $y \in C\left[I, R^{1}\right]$, by direct computation,

$$
\begin{aligned}
|A x(t)-A y(t)| & =\left|\int_{0}^{t} e^{M(s-t)}[R T(y(s)-x(s))+Q S(y(s)-x(s))] d s\right| \\
& \leq K \int_{0}^{t}|x(s)-y(s)| d s .
\end{aligned}
$$

Take $\alpha=\beta=0$ and $K=R\|T\|+Q\|S\|$, by Theorem 2.1, we conclude that $A$ has a unique fixed point in $C\left[I, R^{1}\right]$, that is, (3.3) has exactly one solution in $C^{1}\left[I, R^{1}\right]$.

Thus, for $\eta(t)=p(t)$, then (3.3) has exactly one solution $x_{p}(t)$ in $C^{1}\left[I, R^{1}\right]$, which is the lower solution of (3.2). Set $p_{1}(t)=x_{p}(t)$. By the comparison principle of ordinary differential equations, we have $p(t) \leq p_{1}(t)$. Similarly, for $\eta(t)=p_{1}(t)$, we obtain the unique solution $p_{2}(t)$ of (3.3) with $p_{1}(t) \leq p_{2}(t)$. Continuing this process, we obtain an increasing sequence $\left\{p_{n}\right\} \subset C^{1}\left[I, R^{1}\right]$ such that all $p_{n}(t)(n=0,1, \ldots)$ are the lower solutions of (3.2). Similarly, starting from $q(t)$, we obtain a decreasing sequence $\left\{q_{n}\right\} \subset C^{1}\left[I, R^{1}\right]$ such that all $q_{n}(t)(n=0,1, \ldots)$ are the upper solutions of (3.2). Then it is easy to obtain the conclusions of Theorem 3.4. This completes the proof.

REMARK 3.5. In Theorem 3.4, we may remove the assumption

$$
\left(R k_{0}+Q h_{0}\right)\left(e^{M}-1\right) \leq M, \quad Q h_{0}\left(e^{M}-1\right)<M,
$$

which is necessary in [5], and $k_{0}=\max \{k(t, s) \mid(t, s) \in \Omega\}, h_{0}=\max \{h(t, s) \mid$ $\left.(t, s) \in I^{2}\right\}$. Thus, our new results are more effective than the previous results.

\section{References}

[1] H. Covitz and S. B. Nadler, 'Multivalued contraction mappings in generalized metric spaces', Israel J. Math. 8 (1970), 5-11.

[2] E. de Pascale and L. de Pascale, 'Fixed points for some non-obviously contractive operators', Proc. Amer. Math. Soc. 130 (2002), 3249-3254.

[3] E. de Pascale and P. P. Zabreiko, 'Fixed point theorems for operators in spaces of continuous functions', Fixed Point Theory 5 (2004), 117-129. 
[4] Y. Liu, J. Wu and Z. Li, 'Common fixed points of singlevalued and multivalued maps', Int. J. Math. Math. Sci. 19 (2005), 3045-3055.

[5] B. Lou, 'Fixed points for operators in a space of continuous functions and applications', Proc. Amer. Math. Soc. 127 (1999), 2259-2264.

[6] T. Suzuki, 'Lou's fixed point theorem in a space of continuous mappings', J. Math. Soc. Japan. 58 (2006), 669-774.

JUN WU, College of Mathematics and Computer Science, Changsha University of Science Technology, Changsha, 410076, People's Republic of China e-mail: junwmath@hotmail.com

YICHENG LIU, Department of Mathematics and System Sciences, College of Science, National University of Defense Technology, Changsha, 410073, People's Republic of China

e-mail: liuyc2001@ hotmail.com 